\title{
Small intestinal metabolism is central to whole-body insulin resistance
}

\author{
Giulia Angelini (D) ${ }^{1,2}$ Serenella Salinari, ${ }^{3}$ Lidia Castagneto-Gissey, ${ }^{4}$ \\ Alessandro Bertuzzi, ${ }^{5}$ James Casella-Mariolo, ${ }^{6}$ Sofie Ahlin, ${ }^{7}$ Ivo Boskoski (D) , ${ }^{1,2}$ \\ Melania Gaggini, ${ }^{8}$ Marco Raffaelli, ${ }^{1,2}$ Guido Costamagna, ${ }^{1,2}$ Giovanni Casella, ${ }^{4}$ \\ Pier Luigi Marini, ${ }^{6}$ Amalia Gastaldelli (D) , ${ }^{8}$ Stefan Bornstein, 9,10 \\ Geltrude Mingrone (1) 1,2,9
}

- Additional material is published online only. To view, please visit the journal online (http://dx.doi.org/10.1136/ gutjn-2020-322073)

For numbered affiliations see end of article.

\section{Correspondence to}

Professor Geltrude Mingrone, Internal Medicine, Universita Cattolica del Sacro Cuore, Rome 00168, Italy;

geltrude.mingrone@unicatt.it

GA and SS are joint first authors.

$\mathrm{SB}$ and $\mathrm{GM}$ are joint senior authors.

Received 3 June 2020 Revised 14 July 2020 Accepted 24 July 2020 Published Online First 29 September 2020

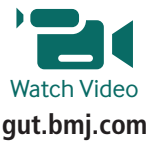

\section{Linked}

- http://dx.doi.org/10.1136/ gutjnl-2020-322662

Check for updates

(C) Author(s) (or their employer(s)) 2021. No commercial re-use. See rights and permissions. Published by BMJ.

To cite: Angelini $\mathrm{G}$,

Salinari S, Castagneto-

Gissey L, et al. Gut

2021:70:1098-1109.

\section{ABSTRACT}

Objective To assess the role of jejunum in insulin resistance in humans and in experimental animals. Design Twenty-four subjects undergoing biliopancreatic diversion (BPD) or Roux-en-Y gastric bypass (RYGB) were enrolled. Insulin sensitivity was measured at baseline and at 1 week after surgery using oral glucose minimal model. We excluded the jejunum from intestinal continuity in pigs and created a jejunal loop with its vascular and nerve supply intact accessible from two cutaneous stomas, and reconnected the bowel with an end-to-end anastomosis. Glucose stable isotopes were given in the stomach or in the jejunal loop.

In vitro studies using primary porcine and human hepatocytes or myoblasts tested the effects of plasma on gluconeogenesis or glucose uptake and insulin signalling. Results Whole-body insulin sensitivity $\left(S \cdot 10^{4}\right.$ :

$0.54 \pm 0.12$ before vs $0.82 \pm 0.11$ after $B P D, p=0.024$ and $0.41 \pm 0.09$ before vs $0.65 \pm 0.09 / \mathrm{pM} / \mathrm{min}$ after RYGB, $\mathrm{p}=$ not significant) and Glucose Disposition Index increased only after BPD. In pigs, insulin sensitivity was significantly lower when glucose was administered in the jejunal loop than in the stomach (glucose rate of disappearance $\left(R_{d}\right)$ area under the curve (AUC)/insulin AUC $\cdot 10: 1.82 \pm 0.31$ vs $2.96 \pm 0.33 \mathrm{mmol} / \mathrm{pM} / \mathrm{min}$, $\mathrm{p}=0.0017)$.

Metabolomics showed a similar pattern before surgery and during jejunal-loop stimulation, pointing to a higher expression of gluconeogenetic substrates, a metabolic signature of impaired insulin sensitivity.

A greater hepatocyte phosphoenolpyruvatecarboxykinase and glucose-6-phosphatase gene expression was elicited with plasma from porcine jejunal loop or before surgery compared with plasma from jejunectomy in pigs or jejunal bypass in humans. Stimulation of myoblasts with plasma from porcine jejunal loop or before surgery reduced glucose uptake, Ser473-Akt phosphorylation and GLUT4 expression compared with plasma obtained during gastric glucose administration after jejunectomy in pigs or after jejunal bypass in humans.

Conclusion Proximal gut plays a crucial role in controlling insulin sensitivity through a distinctive metabolic signature involving hepatic gluconeogenesis and muscle insulin resistance. Bypassing the jejunum is beneficial in terms of insulin-mediated glucose disposal in obesity.

\section{Significance of this study}

What is already known on this subject?

- Metabolic surgery (MS) is an effective treatment for type 2 diabetes (T2D) that induces diabetes remission and improves glycaemic control both in the short term and in the long term.

- Interestingly, a greater improvement of insulin resistance has been reported for those metabolic procedures that bypass large portions of the jejunum.

- In spite of a huge effort aiming at elucidating the mechanisms of action of MS on insulin sensitivity and T2D, a consensus has not been reached yet.

What are the new findings?

- We show that participants exhibited increased whole-body insulin sensitivity and Glucose Disposition Index early after biliopancreatic diversion (BPD).

- Using stable isotopes in pigs, we found that insulin sensitivity deteriorated when glucose was administered in the jejunal loop as compared with the intragastric administration.

- Metabolomics showed a similar metabolic pattern in the obesity state and during jejunalloop glucose stimulation, pointing to an increased gluconeogenesis rate.

- In vitro studies demonstrated greater levels of rate-limiting enzymes for gluconeogenesis in primary cultures of hepatocytes and impaired insulin signalling in myoblasts when incubated with plasma from porcine jejunal loop or human obesity state as compared with plasma from jejunectomy in pigs or jejunal bypass in humans.

- The jejunum plays a crucial role in controlling insulin sensitivity in both animals and humans.

Trial registration number NCT03111953.

\section{INTRODUCTION}

Metabolic surgery (MS) is an effective treatment for type 2 diabetes (T2D) that induces diabetes 
Significance of this study

How might it impact on clinical practice in the foreseeable future?

- In clinical practice, BPD may be superior to Roux-en-Y gastric bypass with respect to improving glycaemic control in patients with marginal endocrine pancreatic function at baseline as it is not reliant on boosting insulin secretion. Our results lay the foundation for the discovery of gut molecular mechanisms implicated in the pathophysiology of insulin resistance and, thus, to new possible drugs for the treatment of T2D mimicking the effects of bariatric/MS.

remission or, at least, significantly improves glycaemic control both in the short term ${ }^{1-3}$ and in the long term. ${ }^{4-7}$ Insulin resistance, which is the major driver of T2D, manifesting long before $\beta$-cell failure develops, ${ }^{8}$ is normalised shortly after MS when body weight is not significantly reduced. ${ }^{9}{ }^{10}$

In spite of a huge effort aiming at elucidating the mechanisms of action of MS on insulin sensitivity and T2D, a consensus has not been reached yet. ${ }^{11-17}$

Hyperplasia of the alimentary limb has been described in rodents undergoing Roux-en-Y gastric bypass (RYGB). ${ }^{18}$ More recently, reprogramming of intestinal glucose metabolism toward an increased glucose consumption was observed in the alimentary Roux limb of both rats and humans. ${ }^{19}$ No data are, however, available at this regard on the alimentary limb features in biliopancreatic diversion (BPD), that is, whether it can work as a postprandial glucose sink similarly to what happens after RYGB or not.

Interestingly, the longer the portion of the jejunum bypassed during MS, the larger is the improvement of insulin sensitivity. ${ }^{15}$ In corpulent and diabetic Goto-Kakizaki rats or high-fat diet fed rats, duodenal-jejunal bypass or jejunectomy improve insulin sensitivity ${ }^{20}$ without changes in incretin circulating levels. ${ }^{21}$ In humans, surgical procedures that exclude shorter or longer portions of the upper gastrointestinal tract, like BPD or RYGB, fully normalise or improve insulin sensitivity few days after the operation and show greater T2D remission when compared with restrictive procedures. ${ }^{22}$ Moreover, T2D remission rates are greater after BPD than after RYGB. ${ }^{2-4}$ Similar to the early effects of BPD and RYGB surgery, severe caloric restriction in individuals affected by obesity leads also to a rapid improvement of glucose control. ${ }^{23}$

We recently showed that even when subjects with obesity display the same weight reduction, specifically $20 \%$ of their baseline weight, whole-body insulin sensitivity is improved more after BPD than after RYGB using either a mixed meal with glucose stable isotopes or a euglycaemic hyperinsulinaemic clamp. $^{15}$

Given that the major difference between RYGB and BPD derives from the bypass of the jejunum in the latter, it is conceivable that the jejunum secretes factors responsible for insulin resistance onset. Accordingly, an acute infusion of nutrients directly into the distal jejunum significantly improves insulin sensitivity in subjects with normal glucose tolerance or with T2D. ${ }^{24}$

Hence, we postulated that the jejunum takes a crucial role in the development of insulin resistance and, therefore, we assessed the role of jejunum in insulin resistance both in humans and in experimental animals.

In humans, we studied glucose kinetics and insulin secretion before and at 1 week after RYGB or BPD in subjects with obesity and insulin resistance. While in RYGB only the duodenum is bypassed, in BPD, the duodenum and the entire jejunum are excluded from food transit.

In pigs, we excluded the jejunum from the intestinal continuity and attached its proximal and distal ends to the skin, while the remaining bowel was reconnected with an end-to-end anastomosis. In this way, it was possible to obtain a jejunal loop with its vascular and nerve supply intact, the so-called Thiry-Vella loop (TRVL), which could be infused selectively with $\mathrm{U}^{13} \mathrm{C}$-glucose, while $6,6^{2} \mathrm{H}_{2}$-glucose was infused intravenously to assess the modification of insulin sensitivity. In vitro studies investigated the role of circulating factors in hepatic gluconeogenesis and muscle insulin signalling.

\section{METHODS}

\section{Human studies}

\section{Subjects}

Sample size was calculated in a previous study in which we measured insulin sensitivity at $20 \% \mathrm{wt}$ reduction. ${ }^{15}$

Twenty-four subjects with obesity and insulin resistance but normal glucose tolerance were studied with an oral glucose tolerance test (OGTT) before and at 1 week after BPD $(n=12)$ or RYGB $(n=12)$.

Inclusion and exclusion criteria have been previously reported. ${ }^{15}$

\section{Metabolic surgery}

BPD (online supplementary figure 1) and RYGB (online supplementary figure 2 ) procedures have been previously described. ${ }^{15}$

\section{Oral glucose tolerance test}

Participants were admitted to the ward in the afternoon of the day preceding the OGTT. At 18:00, they received a standard meal (12 kcal/kg lean body mass) consumed by 19:00. After this meal, participants fasted, except for water, until completion of the study the following day. At 07:00, a catheter was inserted into an antecubital vein of one arm to obtain blood samples at $15,30,45,60,80,100,120,140,160,180,200,220,240,260$, $280,300,320,340$ and $360 \mathrm{~min}$ to measure glucose, insulin and C-peptide.

For glucagon-like peptide 1 (GLP-1) analyses, blood samples $(5 \mathrm{~mL})$ were drown in EDTA and aprotinin (400 kallikrein inhibitor units/mL blood; Bayer, Leverkusen, DE) tubes every 20-360 min and kept on ice to avoid hormone degradation.

\section{Animal studies}

Pig operation

Experimental procedures were performed at the Animal House of the Catholic University in Rome, Italy, after approval from the ethical committee for animal studies. Four healthy adult pigs weighing $52 \pm 8 \mathrm{~kg}$ (Landrace Breeding) were used for the study. All surgical procedures were performed under general anaesthesia after an overnight fast.

The TRVL (online supplementary figure 3 ) was performed by isolating $1 \mathrm{~m}$ of jejunum with its vascular and nerve supply intact and exteriorising the two open ends by means of two cutaneous stomas. The TRVL was exteriorised on ventral portion where the muscle layer is thinner in order to prevent intestinal ischaemia. The end of the duodenum was sutured in an end-to-end fashion to the proximal end of the transectum ileum.

\section{Measurement of glucose disposal}

One week after jejunectomy and TRVL were performed, the animals were randomly studied in two different sessions: glucose 
was administered by gastric gavage or though the TRVL. The other study was performed at a distance of 24 hours.

At 0800 hours, $\left[6,6-{ }^{2} \mathrm{H}_{2}\right.$ ]glucose was infused (priming dose: $22 \mu \mathrm{mol} / \mathrm{kg}$ prime; infusion rate: $0.22 \mu \mathrm{mol} / \mathrm{kg} / \mathrm{min}$ ) to determine endogenous glucose kinetics. After 2 hours of isotope infusion (basal period), $75 \mathrm{~g}$ glucose dissolved in saline solution was injected over $5 \mathrm{~min}$ in the stomach via a gastric catheter positioned endoscopically under anaesthesia or through the TRVL. The intragastric glucose load was enriched with $0.9 \mathrm{~g}$ of $\left[\mathrm{U}^{13} \mathrm{C}\right]$-glucose tracer. Blood glucose and insulin were measured basally, at $5 \mathrm{~min}$ and thereafter every 10-180 $\mathrm{min}$.

For GLP-1 analyses, blood samples $(5 \mathrm{~mL})$ were drawn at $0,30,60,90,120,150$ and $180 \mathrm{~min}$ in EDTA and aprotinin (400 kallikrein inhibitor units/mL blood; Bayer, Leverkusen, Germany) tubes kept on ice to avoid hormone degradation.

Assays in humans and pigs as well as in vitro study details are reported in the online supplementary material.

\section{Mathematical models}

Whole-body insulin sensitivity after the OGTTs in humans, or after intragastric or intra-TRVL glucose administration in pigs, was computed using the equations of the oral glucose minimal model $^{25}$ :

$$
\begin{aligned}
& \frac{d}{d t} G(t)=-S_{G}\left(G(t)-G_{b}\right)-S_{I} Z(t) G(t)+\frac{R a(t)}{V_{G}}, G(0)=G_{b} \\
& \frac{d}{d t} Z(t)=-p Z(t)+p\left(I(t)-I_{b}\right), Z(0)=0
\end{aligned}
$$

where $G(t)$ and $I(t)$ are, respectively, the glucose and insulin concentrations in plasma, with $G_{b}$ and $I_{b}$ as their baseline values, and $Z(t)$ is a variable accounting for the delay in insulin action. The profile of the rate of appearance of orally ingested glucose $\left(\mathrm{R}_{\mathrm{a}}\right)$ was obtained from the stable isotopes analysis. Glucose effectiveness $\left(S_{G}\right)$, insulin sensitivity $\left(S_{I}\right)$, rate constant of insulin action $(p)$ and glucose distribution volume $\left(V_{G}\right)$ were computed by means of Eqs. (1) and (2) using glucose and insulin concentration data.

The profile of the insulin secretion rate (ISR) and the indexes of $\beta$-cell sensitivity to glucose (the dynamic $\beta$-cell glucose sensitivity, $\Phi_{d}$, the static sensitivity, $\Phi_{s}$, plus the total sensitivity $(\Phi)$ were computed by the C-peptide minimal model as proposed by Breda et al. ${ }^{26}$

The parameters of the glucose and C-peptide minimal models were estimated by minimisation of a weighted least-squares index using a constrained Levenberg-Marquardt minimisation routine of the MATLAB library. The SEs of the estimates of individual parameters were evaluated by the linearisation method, and the coefficients of variation were found to be $<20 \%$.

\section{Calculations}

The areas under the curves (AUCs) during the OGTT were calculated using the trapezoidal rule. The incremental ISR AUC was computed by subtracting the basal value. Insulin clearance was calculated as $\mathrm{ICR}=\left(\mathrm{ISR}_{\mathrm{AUC}}\right.$ insulin $\left._{\mathrm{AUC}}\right)-\left(\mathrm{V} \times\left[\right.\right.$ (insulin end time $^{-}$insu$\left.\operatorname{lin}_{\text {start time }}\right)$ /insulin $\left.{ }_{\mathrm{AUC}} \mathrm{J}\right)$, where $\mathrm{V}$ is the insulin volume of distribution assumed to be $0.14 \mathrm{~L} / \mathrm{kg} .{ }^{27}$ In pigs, insulin clearance was calculated as the ratio of C-peptide AUC to insulin AUC.

\section{Statistical analysis}

We used non-parametric tests because of the relatively small number of subjects and because many variables were not normally distributed. To compare outcomes between RYGB and BPD groups, we used the Mann-Whitney U test.

The Wilcoxon signed-rank test was used to detect differences between variables before and after interventions within the same groups and was Bonferroni corrected for multiple comparisons. Repeated-measures analysis of variance was used to compare plasma levels of GLP-1 before and after interventions. Data are expressed as the mean \pm SEM unless otherwise specified. Statistical significance was set at $\mathrm{p}<0.05$ (two-tailed).

Stochastic optimisation of the Glucose Disposition Index curve fitting was obtained by the Monte Carlo method.

We performed metabolomic statistical analysis using MetaboAnalyst web tool (www.metaboanalyst.ca). We checked for data integrity and missing value using Singular Value Decomposition (SVDIMPUTE) algorithm. Data were log transformed and scaled using mean centring algorithm.

Principal component analysis (PCA) model with permutation testing algorithm was used to visualise and compare metabolite profiles and to detect metabolic variation between and among

Table 1 Insulin sensitivity and insulin secretion, measured by the oral glucose minimal model, and insulin clearance before and at 1 week after

\begin{tabular}{|c|c|c|c|c|c|c|}
\hline & \multicolumn{3}{|l|}{ BPD } & \multicolumn{3}{|l|}{ RYGB } \\
\hline & Before & After 1 week & $P$ value & Before & After 1 week & $P$ value \\
\hline $\mathrm{S}_{\mathrm{G}} \cdot 10^{2}$ (per min) & $1.95 \pm 0.25$ & $2.88 \pm 0.22 \dagger \neq \S \emptyset$ & 0.030 & $1.76 \pm 0.34$ & $3.00 \pm 0.31$ & NS \\
\hline $\mathrm{S}_{1} \cdot 10^{4}(/ \mathrm{pM} / \mathrm{min})$ & $0.54 \pm 0.12$ & $0.82 \pm 0.11$ & 0.024 & $0.41 \pm 0.09$ & $0.65 \pm 0.09$ & NS \\
\hline $\mathrm{p} \cdot 10^{2}$ (per min) & $0.44 \pm 0.15$ & $0.22 \pm 0.08$ & NS & $0.43 \pm 0.10$ & $0.14 \pm 0.01$ & 0.015 \\
\hline Volume (litre) & $26.67 \pm 4.58$ & $25.46 \pm 3.90$ & 0.001 & $24.79 \pm 3.32$ & $24.41 \pm 3.22$ & 0.012 \\
\hline HOMA-IR & $5.29 \pm 0.71$ & $1.61 \pm 0.40$ & 0.0002 & $5.91 \pm 0.43$ & $2.46 \pm 0.39$ & 0.001 \\
\hline$\Phi_{s} \cdot 10^{9}$ (per min) & $44.69 \pm 5.83$ & $38.03 \pm 4.67$ & NS & $45.38 \pm 3.51$ & $38.65 \pm 5.22$ & NS \\
\hline$\Phi_{D} \cdot 10^{9}$ & $530.0 \pm 204.2$ & $412.5 \pm 146.6$ & NS & $1108.6 \pm 221.03$ & $1223.4 \pm 247.7$ & NS \\
\hline$\Phi \cdot 10^{9}$ (per min) & $52.83 \pm 5.64$ & $58.18 \pm 11.38$ & NS & $67.48 \pm 7.41$ & $60.55 \pm 5.76$ & NS \\
\hline $\mathrm{ISR}_{\mathrm{AUC}}(\mathrm{nmol})$ & $99.18 \pm 11.71$ & $70.86 \pm 10.40$ & 0.021 & $74.98 \pm 6.00$ & $111.43 \pm 20.24$ & 0.011 \\
\hline $\begin{array}{l}\text { Insulin clearance } \\
(\mathrm{L} / \mathrm{min})\end{array}$ & $0.71 \pm 0.07$ & $1.73 \pm 0.29$ & 0.002 & $0.60 \pm 0.05$ & $1.45 \pm 0.17$ & 0.001 \\
\hline
\end{tabular}
metabolic surgery

Data are expressed as mean \pm SEM

Volume indicates glucose volume of distribution.

$\Phi$, total $\beta$-cell glucose sensitivity; BPD, biliopancreatic diversion; $\Phi_{D^{\prime}}$ dynamic $\beta$-cell glucose sensitivity; HOMA-IR, homeostatic model assessment of insulin resistance; ISR ${ }_{\text {AUc }}$ incremental area under the curve of the insulin secretion rate over basal; NS, not significant; $p$, minimal model parameter; RYGB, Roux-en-Y gastric bypass; $\Phi$, static $\beta$-cell glucose sensitivity; $S_{G}$ glucose effectiveness; $S_{1}$, insulin sensitivity; Volume, glucose volume of distribution. 
A

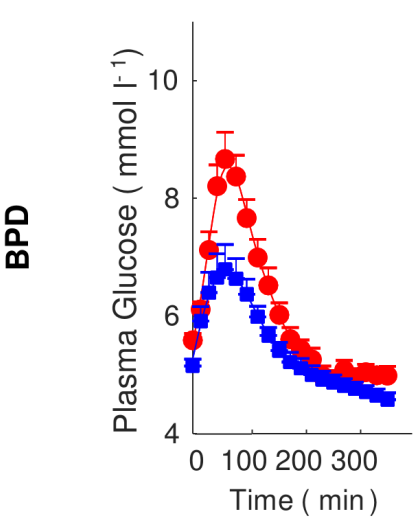

B

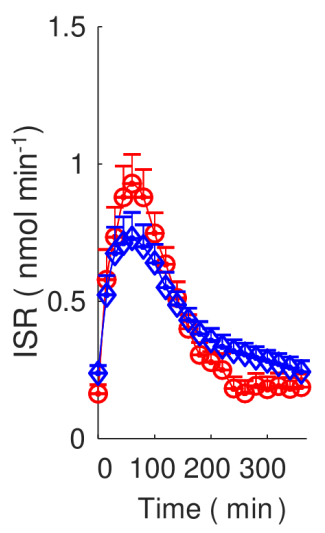

C

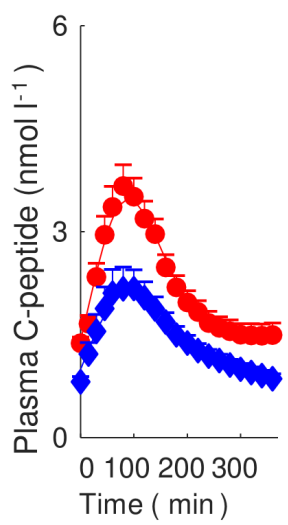

D

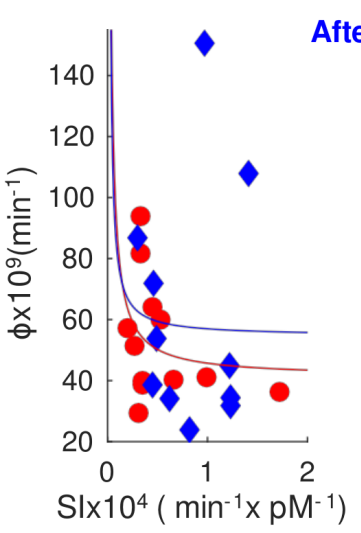

Before Surgery

After Surgery
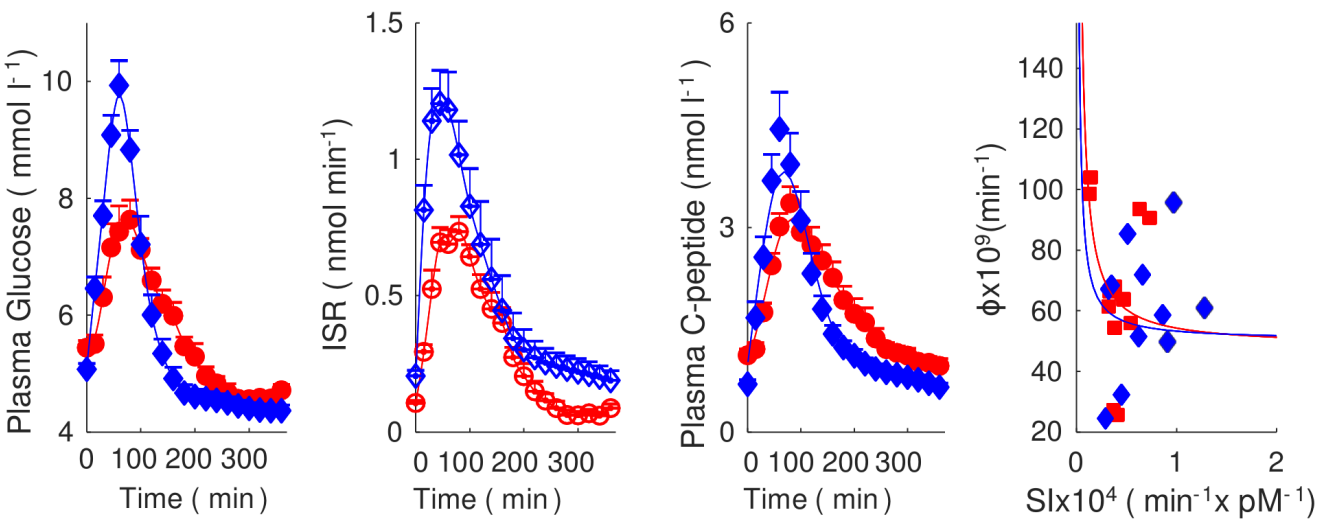

Figure 1 Glucose, insulin and C-peptide time courses before and after surgery. (A-C) Glucose, ISR and C-peptide time courses before and at 1 week after BPD (upper panels) or RYGB (lower panels), respectively. Closed circles and rhombi represent experimental data, while open symbols are estimated values. Continuous lines represent optimal fitting of data by oral glucose (A) and C-peptide (C) minimal models. (D) Glucose Disposition Index values together with the fitting curves before surgery and at 1 week after either BPD (upper panel) or RYGB (lower panel). Total $\beta$-cell glucose sensitivity $(\Phi)$ values are reported on the $y$-axes, while whole-body insulin sensitivity $\left(S_{1}\right)$ values are reported on the $x$-axes. Significance $(p<0.05)$ between curves was assessed by repeated-measures analysis of variance and reported as follows: plasma glucose before versus after BPD: $0,30,45$, $60,80,100,120,140,160,340$ and $360 \mathrm{~min}$; plasma glucose before versus after RYGB: $0,15,30,45,60,80,140,160,180,200,220$ and 360 min; plasma C-peptide before versus after BPD: 0, 15, 30, 45, 60, 80, 100, 120, 140, 160, 180, 220, 240, 260, 280, 300, 320, 340 and 360 min; plasma Cpeptide before versus after: RYGB: $0,15,30,45,60,140,160,180,200,220,240,260,280,300,320,340$ and 360 min. Colour legend: red before MS and blue after MS. BPD, biliopancreatic diversion; ISR, insulin secretion rate; MS, metabolic surgery; RYGB, Roux-en-Y gastric bypass.

groups. To determine the number of factors to extract, a scree plot (online supplementary figures 4 and 5) was used. The univariate statistical analysis was used to perform the intergroup comparisons.

The heat map was used as a graphical representation of metabolomic average values per experimental categories, including only metabolites for which the procedure interaction from the mixed-effect analyses was significant.

Changes in metabolite levels were calculated as the $\log _{2}$ fold change (FC) ratio and graphically depicted by box plots.

The Kruskal-Wallis test was used for between-group comparisons, while the Wilcoxon test was used to detect within-group differences.

To identify the metabolic pathways involved, the validated metabolites were annotated with Human Metabolome Database (http://www.hmdb.ca/).

\section{RESULTS}

\section{Improvement of insulin sensitivity after MS in humans}

To test the early effects of the jejunal bypass action on the improvement of insulin resistance, we studied 24 subjects undergoing RYGB $(n=12)$ or BPD $(n=12)$ at baseline and at 1 week after surgery.

The subjects were matched by age $(43.9 \pm 2.3$ and $40.7 \pm 3.1$ years in BPD and RYGB groups, respectively; $\mathrm{p}=0.120)$, by basal plasma glucose $(5.63 \pm 0.13$ vs $5.46 \pm 0.11 \mathrm{mmol} / \mathrm{L}$ in $\mathrm{BPD}$ vs RYGB; $\mathrm{p}=0.820)$ and insulin $(172.2 \pm 27.6$ vs $159.6 \pm 16.8$ vs $\mathrm{pmol} / \mathrm{L}$ in $\mathrm{BPD}$ vs $\mathrm{RYGB} ; \mathrm{p}=0.140$ ).

In the BPD group, baseline weight was $163.1 \pm 7.8 \mathrm{~kg}$ and $161.2 \pm 7.5 \mathrm{~kg}$ at 1 week after surgery $(\mathrm{p}=0.001)$; in the RYGB group, baseline weight was $148.3 \pm 6.3 \mathrm{~kg}$ and $145.9 \pm 5.9 \mathrm{~kg}$ at 1 week after surgery $(\mathrm{p}=0.003)$. The weight reduction was similar in the two groups $(-1.1 \pm 0.8 \%$ after BPD and $-1.5 \pm 1.2 \%$ after RYGB, $p=0.349$ ).

In subjects who underwent BPD and, thus, had the jejunal bypass, whole-body insulin sensitivity $\left(\mathrm{S}_{\mathrm{I}}\right)$ was almost doubled at 1 week after surgery (table 1 ). Instead, insulin sensitivity tended to improve without reaching statistical significance after RYGB (table 1).

Total insulin secretion (ISR $\mathrm{AUC}_{\mathrm{AC}}$ ) was significantly increased only after RYGB (table 1). 
Table 2 Upper part: minimal model analysis of glucose, insulin and C-peptide time courses following glucose administration via gastric gavage after jejunectomy or in the TRVL. Lower part: stable isotope labelled glucose kinetics.

\begin{tabular}{|c|c|c|c|}
\hline & $\begin{array}{l}\text { TRVL glucose } \\
\text { administration }\end{array}$ & $\begin{array}{l}\text { Gastric glucose } \\
\text { administration }\end{array}$ & $P$ value \\
\hline \multicolumn{4}{|l|}{ Glucose minimal model } \\
\hline $\mathrm{S}_{\mathrm{G}} \cdot 10^{2}$ (per $\mathrm{min}$ ) & $2.22 \pm 0.83$ & $2.94 \pm 0.81$ & NS \\
\hline $\mathrm{p} \cdot 10^{2}$ (per min) & $0.16 \pm 0.03$ & $0.14 \pm 0.02$ & NS \\
\hline Volume (litre) & $5.26 \pm 0.44$ & $5.25 \pm 0.44$ & NS \\
\hline $\mathrm{S}_{1} \cdot 10^{4}(/ \mathrm{pM} / \mathrm{min})$ & $1.10 \pm 0.32$ & $3.25 \pm 0.50$ & 0.0062 \\
\hline \multicolumn{4}{|c|}{ Stable isotope-labelled glucose kinetics } \\
\hline $\begin{array}{l}\text { EGP AUC.insulin AUC. } 10^{4} \\
(\mathrm{mmol} \cdot \mathrm{pM} \cdot \mathrm{min})\end{array}$ & $4.42 \pm 0.91$ & $2.57 \pm 0.54$ & 0.028 \\
\hline $\begin{array}{l}\mathrm{R}_{\mathrm{d}} \mathrm{AUC} / \mathrm{insulin} \text { AUC } 10 \\
(\mathrm{mmol} / \mathrm{pM} / \mathrm{min})\end{array}$ & $1.82 \pm 0.31$ & $2.96 \pm 0.33$ & 0.0017 \\
\hline $\begin{array}{l}\text { Insulin clearance } \\
\text { C-peptide AUC/insulin AUC/102 }\end{array}$ & $2.13 \pm 0.50$ & $3.52 \pm 0.43$ & NS \\
\hline $\begin{array}{l}\text { C-peptide AUC } \cdot 10^{4} \\
\text { (nM-min) }\end{array}$ & $1.03 \pm 0.27$ & $1.56 \pm 0.35$ & NS \\
\hline
\end{tabular}

Data are expressed as mean \pm SEM.

$A U C$, area under the curve; EGP, endogenous glucose production; NS, not significant; $R_{d^{\prime}}$ rate of glucose disappearance; TRVL, Thiry-Vella loop.

Figure 1A-C shows plasma glucose, ISR and plasma C-peptide time courses before and after surgery.

Figure 1D depicts the Glucose Disposition Index after BPD or RYGB; the hyperbolic curve was shifted upward in the subjects undergoing BPD as compared with the curve of the subjects who underwent RYGB $(\mathrm{p}<0.01)$. This finding shows that the incremental insulin secretion is reduced after BPD $(99.18 \pm 11.71$ vs $70.86 \pm 10.40 \mathrm{nmol}, \mathrm{p}=0.021$ ) because of the improvement of whole-body insulin sensitivity and $\beta$-cell glucose sensitivity, whereas it is increased after RYGB (74.98 \pm 6.00 vs $111.43 \pm 20.24 \mathrm{nmol}, \mathrm{p}=0.011$ ),

Hepatic insulin sensitivity improved in both groups as shown by the significant reduction of hepatic insulin resistance and the significant increase of insulin clearance.

Homeostatic model assessment of insulin resistance, which is a measure of hepatic insulin resistance, ${ }^{28}$ improved significantly after both BPD $(p=0.0002)$ and RYGB $(p=0.001)$.

Insulin is cleared mainly by the liver, and its clearance is impaired in hepatic insulin resistance. ${ }^{29}$

Accordingly, insulin clearance (table 1) was increased from baseline in both groups $(p=0.002$ for BPD and $p=0.001$ for RYGB).

\section{Glucose injection in the TRVL impairs insulin sensitivity in pigs}

To assess the role of the jejunum in the development of insulin resistance, insulin sensitivity and glucose kinetic were evaluated following intragastric glucose administration as well as during glucose injection in the TRVL.

When glucose was injected in the stomach following jejunectomy, insulin sensitivity was significantly higher in comparison with the glucose administration in the TRVL $(3.25 \pm 0.50$ vs $1.10 \pm 0.32 / \mathrm{pM} / \mathrm{min}, \mathrm{p}=0.0062)($ table 2$)$.
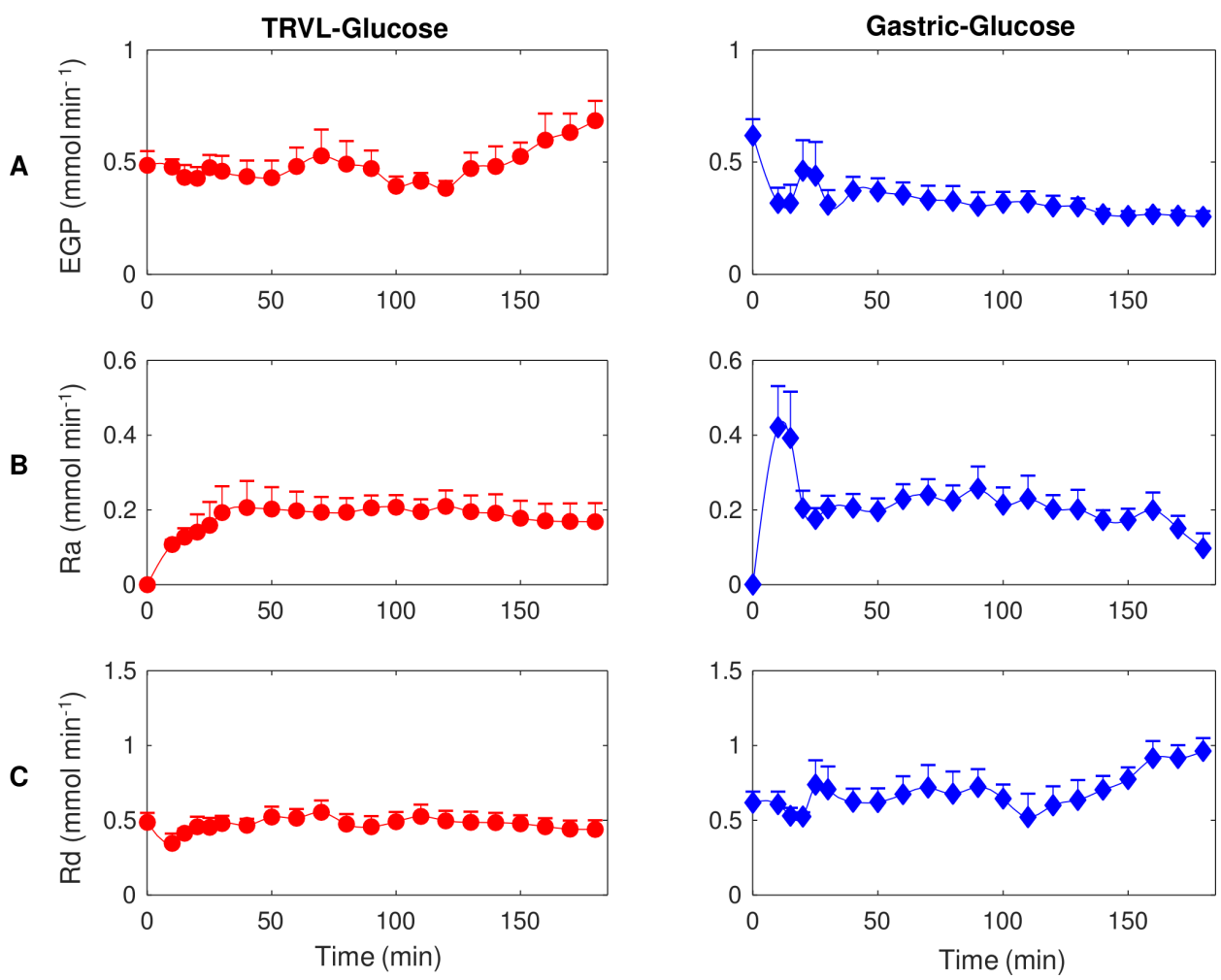

Figure 2 Time courses of the EGP, $R_{a}$ and $R_{d}$. $(A-C) E G P, R_{a}$ and $R_{d}$ measured in pigs after glucose administration in the TRVL (left panels) or gastric glucose administration following jejunectomy (right panels). Significance $(p<0.05)$ between curves was assessed by repeated-measures analysis of variance and reported as follows. EGP TRVL glucose administration versus gastric glucose administration: 5, 40, 160, 170 and 180 min. Rate of glucose appearance $\left(R_{a}\right)$ TRVL glucose administration versus gastric glucose administration: $170 \mathrm{~min}$. Rate of glucose disappearance $\left(R_{d}\right) T R V L$ glucose administration versus gastric glucose administration: 150,160, 170 and 180 min. EGP, endogenous glucose production; $R_{a^{\prime}}$ rate of appearance of exogenous glucose; $R_{d^{\prime}}$ rate of disappearance of exogenous glucose; TRVL, Thiry-Vella loop. 
A

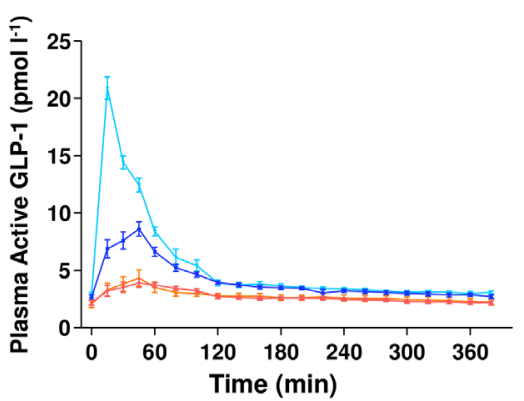

C

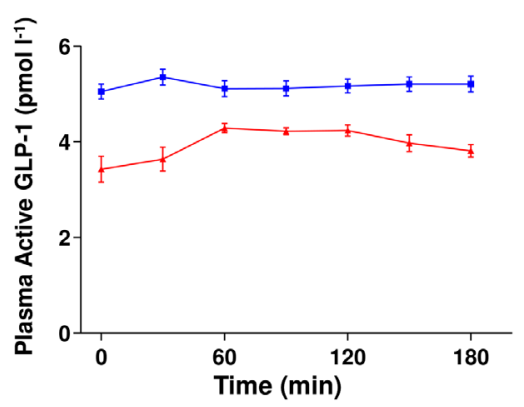

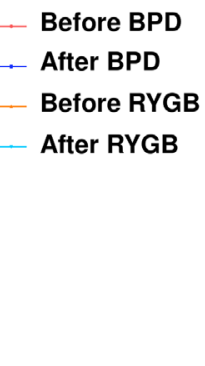

D
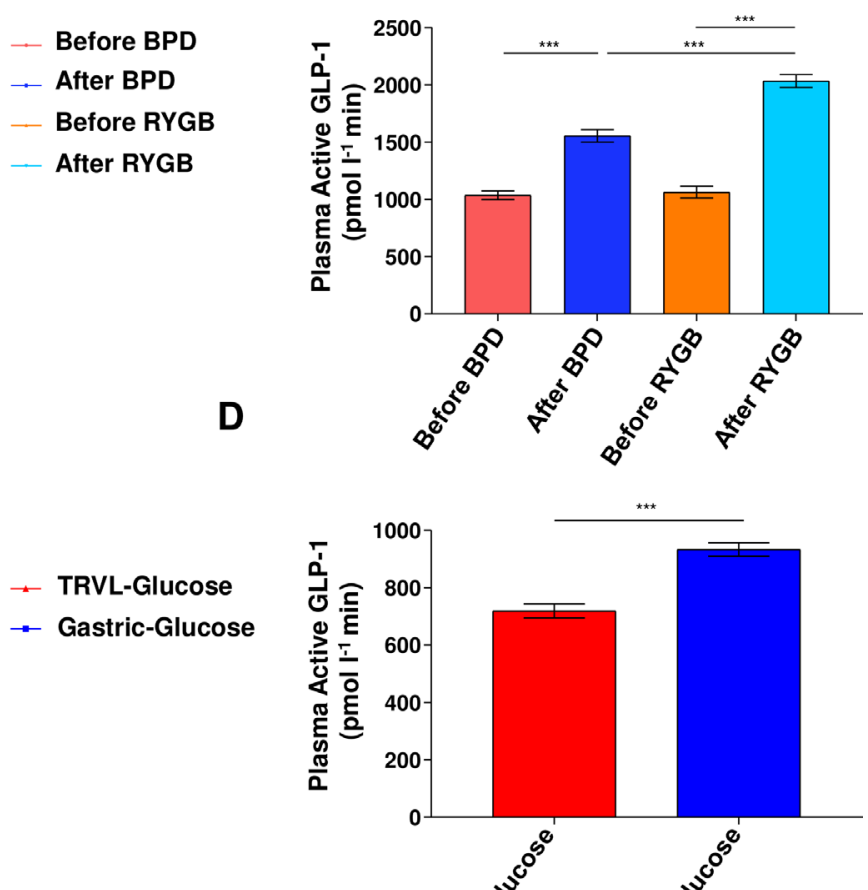

Figure 3 Time courses of active GLP-1. (A) Time courses of active GLP-1 during an oral glucose load before and at 1 week after BPD or RYGB. (B) AUCs of active GLP-1 in humans. (C) Time courses of active GLP-1 concentration measured in pig plasma during glucose administration in the TRVL or via gastric gavage following jejunectomy. (D) AUCs of active GLP-1 in pigs. Significance $(p<0.05)$ between curves was assessed by repeated-measures analysis of variance and reported as follows: before versus after BPD: 30, 45 and 60 min; before versus after RYGB: 15, 30, 45 and 60 min; TRVL glucose administration versus gastric glucose administration: $0,30,60,90,120,150$ and $180 \mathrm{~min} .{ }^{* * *} \mathrm{P}<0.0001$. AUC, area under the curve; $B P D$, biliopancreatic diversion; GLP-1, glucagon-like peptide 1; RYGB, Roux-en-Y gastric bypass; TRVL, Thiry-Vella loop.

The time courses of the endogenous glucose production (EGP), the glucose rate of appearance $\left(\mathrm{R}_{\mathrm{a}}\right)$ and the glucose rate of disappearance $\left(\mathrm{R}_{\mathrm{d}}\right)$ are reported in figure $2 \mathrm{~A}-\mathrm{C}$ in the two experimental conditions (gastric glucose administration vs TRVL). $\mathrm{R}_{\mathrm{a}}$ was not significantly different in the two sets of experiments.

Gastric glucose administration following jejunectomy was associated with a significantly $(p=0.0017)$ higher insulinmediated glucose disappearance rate compared with glucose administration in the TRVL (table 2). Meanwhile, EGP per unit of circulating insulin was significantly higher $(p=0.028)$ when glucose was administered through the TRVL in comparison with gastric administration (table 2).

The insulin clearance rate was higher after gastric glucose administration as compared with the TRVL glucose administration, without reaching a statistical significance (table 2).

Overall, these data show that whole-body insulin-mediated glucose disposal as well as hepatic insulin sensitivity were impaired when glucose was administered in the jejunal TRVL.

\section{GLP-1 time course in humans and pigs}

In humans, GLP-1 increased after both types of surgery in response to the oral glucose challenge, but subjects after RYGB showed a higher plasma concentration than subjects after BPD (figure 3A).

The AUC of active GLP- 1 was $1037 \pm 37.01$ before BPD and $1555 \pm 54.26(\mathrm{pmol} / \mathrm{L}) \cdot \mathrm{min}$ after BPD $(\mathrm{p}<0.0001)$. The GLP-1
AUC was $1064 \pm 51.93$ before vs $2034 \pm 56.25(\mathrm{pmol} / \mathrm{L}) \cdot \mathrm{min}$ after RYGB ( $<0.0001)$ (figure 3B).

GLP-1 circulating levels (figure 3C) were significantly higher when glucose was administered in the stomach as compared with the TRVL glucose load. In fact, it is acknowledged that in pigs, GLP-1 is produced in the distal ileum, cecum and proximal and distal colon. ${ }^{30}$

The AUC of active GLP-1 was $719.4 \pm 24.61$ in the TRVL experiments and $933.8 \pm 23.26(\mathrm{pmol} / \mathrm{L}) \cdot \mathrm{min}$ after gastric glucose administration $(\mathrm{p}<0.0001)$ (figure 3D).

No significant correlations between GLP-1 AUC and measures of insulin sensitivity were found, suggesting that GLP-1 does not cover a major role in insulin sensitivity.

\section{Humans and pigs show similar metabolic signatures jeopardising insulin sensitivity}

To gain further insight into the mechanisms responsible for the improvement of insulin sensitivity after jejunal exclusion, untargeted metabolomics analysis was performed.

We identified 259 metabolites in humans and 264 metabolites in pigs.

In humans, $23.9 \%$ of metabolites changed significantly after BPD.

Figure 4A shows that the first two components of the PCA explain $63.7 \%$ of the variance of those metabolites that significantly changed after MS. 
A Human PCA
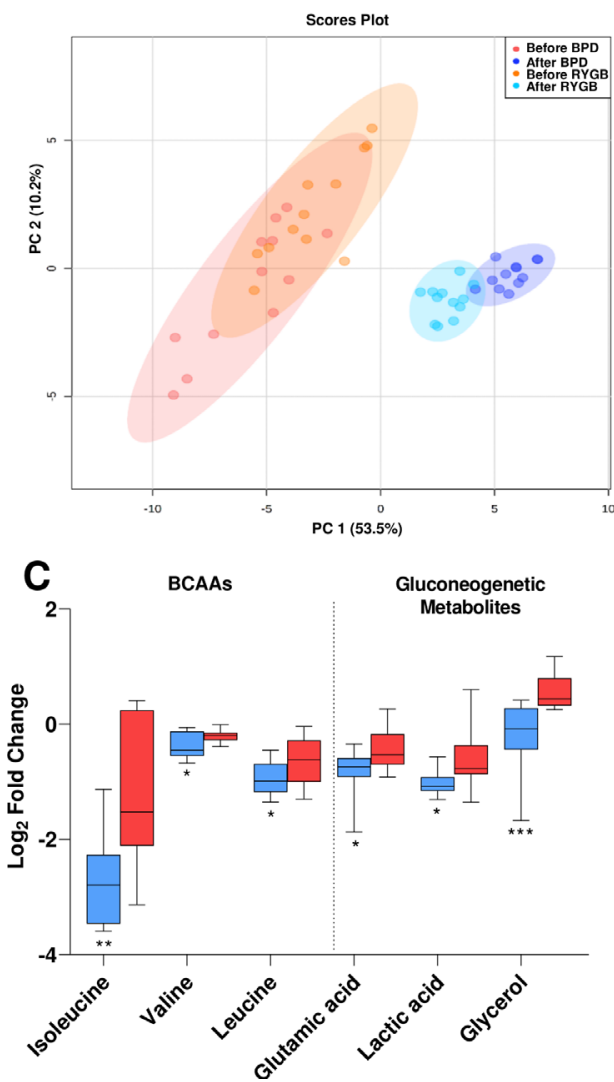

B Human Heatmap

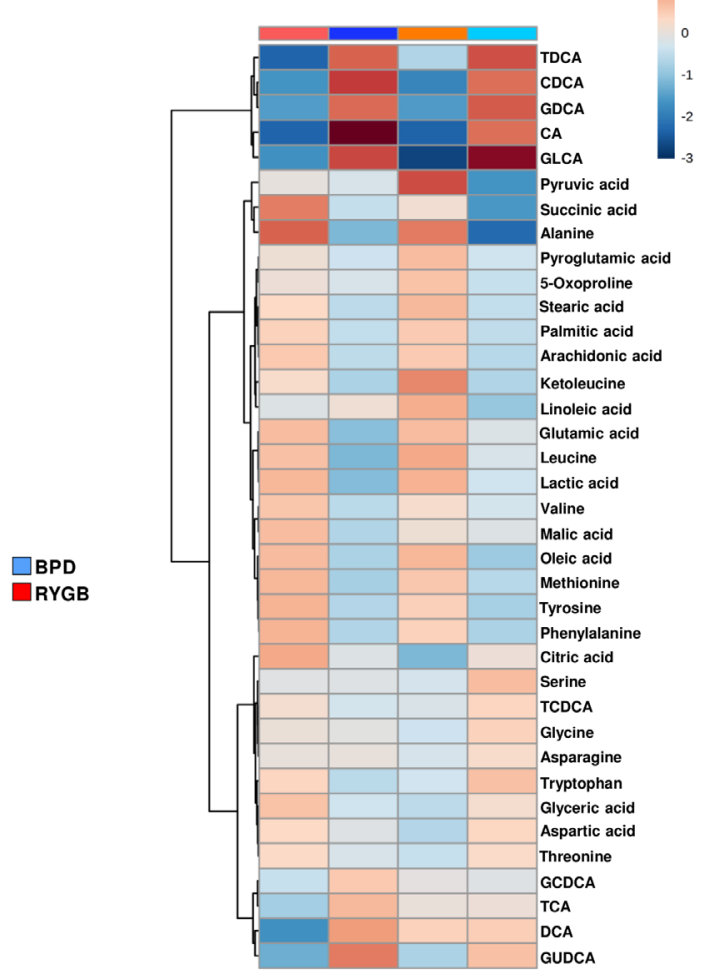

Figure 4 Human metabolomic analysis. (A) PCA of the metabolites before and at 1 week after BPD or RYGB. The explained variances are shown in brackets. (B) Heat map of metabolites before and 1 week after BPD or RYGB. (C) Box plots of median fold change values of the relative abundance of BCAAs and gluconeogenetic metabolites after the OGTT in subjects who have undergone RYGB or BPD. * $p<0.05 ;{ }^{*} \mathrm{P}<0.01 ;{ }^{* *}{ }^{*} \mathrm{P}>0.001$. BCAA, branched-chain amino acid; BPD, biliopancreatic diversion; CA: cholic acid; DCA: deoxycholic acid; GCDCA: glycochenodeoxycholic acid; GDCA: glycodeoxycholic acid; GLCA: glycolithocholic acid; GUDCA: Glycoursodeoxycholic acid; OGTT, oral glucose tolerance test; PCA, principal component analysis; RYGB, Roux-en-Y gastric bypass; TCA, tricarboxylic acid cycle; TCDCA: taurochenodeoxycholic acid; TDCA: taurodeoxycholic acid

The heat maps comparing the levels of metabolites before and after BPD or RYGB (figure 4B) reveal that many metabolites, such as bile acids, proline and 5-oxoproline, increased after MS, particularly in the BPD group. In contrast, other metabolites, like gluconeogenetic substrates (succinic acid, lactic acid and glutamic acid) as well as branched-chain amino acid (BCAA), decreased. Log-transformed mean FC of the relative abundance of BCAAs and gluconeogenetic metabolites after the OGTT in subjects who have undergone RYGB or BPD are depicted as box plots in figure 4C. As expected, these metabolites decreased during the glucose load; however, the decrements were significantly higher after BPD than after RYGB.

In pigs, metabolites significantly different between TRVL and gastric glucose administration were $24.6 \%$.

Components 1 and 2 of the PCA explained $46.6 \%$ of the variance of metabolites significantly differing between TRVL and gastric glucose administration (figure $5 \mathrm{~A}$ ).

Similar to humans, in the porcine heat map (figure 5B), gluconeogenetic precursors and BCAAs were all higher in the TRVL than in the gastric glucose administration experimental condition, while bile acids and proline increased after gastric glucose administration.

These results show a similar metabolic pattern in both human obesity and during the stimulation of jejunal loop with glucose, indicating a higher expression of gluconeogenetic substrates and
BCCAs, a metabolic signature in line with the impaired insulin sensitivity profile.

\section{Plasma from subjects with obesity and from TRVL increases gluconeogenesis in hepatocytes}

To gain further insight into the possible effect of increased plasma gluconeogenetic substrates in obesity and in the jejunal loop experimental condition, we assessed key rate-limiting enzymes of gluconeogenesis in primary hepatocyte cultures incubated with insulin and plasma from humans or pigs.

Compared with plasma from subjects undergoing BPD, plasma of subjects before surgery significantly enhanced PEPCK-1 and G6Pase expression (table 3); no significant changes were observed using plasma of patients undergoing RYGB (table 3) (online supplementary figure 6A,C).

Similar to what observed in human obesity, plasma taken during TRVL experiments increased PEPCK-1 and G6Pase expression when compared with plasma obtained after gastric glucose administration (table 3) (online supplementary figure 6B,D).

These data confirm that a rise in the circulating levels of gluconeogenetic substrates, as observed before MS or in the TRVL experimental condition, promotes liver gluconeogenesis. 


\section{A \\ Porcine PCA \\ B \\ Porcine Heatmap \\ TRVL-Glucose}
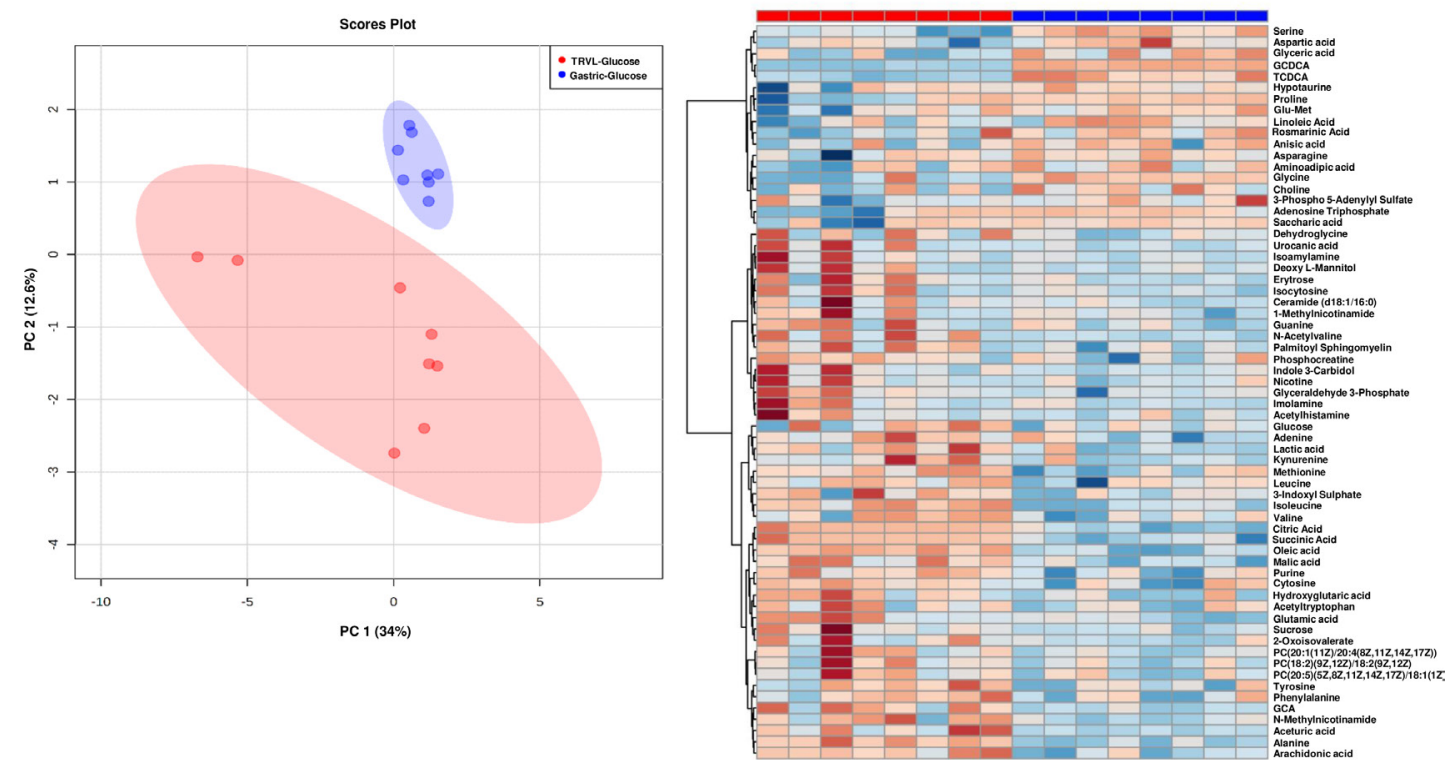

Figure 5 Metabolomic analysis in pigs. (A) PCA explains $46.6 \%$ of the variance of metabolites that significantly differ between TRVL glucose administration versus gastric glucose administration and jejunectomy conditions. (B) Heat map of metabolites during glucose administration in TRVL or via gastric gavage after jejunectomy. GCA, glycocholicacid; GCDCA, glycochenodeoxycholicacid; PCA, principal component analysis; TCDCA, taurochenodeoxycholic acid; TRVL, Thiry-Vella loop.

\section{Plasma from subjects with obesity and from TRVL inhibits Akt phosphorylation and glucose uptake in myoblasts}

To investigate the possible effect of altered plasma metabolic profile on insulin signalling, we used insulin and plasma, obtained from humans or pigs, to stimulate primary myoblast cultures.

Akt Ser473 phosphorylation was significantly increased when myoblasts were stimulated with plasma of subjects undergoing
BPD as compared with presurgical condition (figure 6A), while plasma from subjects who underwent RYGB failed to stimulate Akt phosphorylation (table 3).

A similar increase in Akt Ser473 phosphorylation (figure 6B) was observed when myoblasts were stimulated with plasma taken in pigs during the gastric glucose load after jejunectomy compared with plasma from TRVL experiments (table 3).

Table 3 mRNA expression of gluconeogenesis rate-limiting enzymes (upper part) after in vitro stimulation of primary hepatocyte cultures with insulin and plasma from subjects before and after metabolic surgery (BPD or RYGB or plasma from pigs during glucose administration in the TRVL or during gastric glucose administration); protein expression of Akt phosphorylated on Ser473, GLUT4 and glucose uptake assays (lower part) after in vitro stimulation of primary myoblast cultures with insulin and plasma from humans or pigs (see previous specifications)

\begin{tabular}{|c|c|c|c|c|c|c|c|c|c|}
\hline & $\begin{array}{l}\text { Plasma } \\
\text { before BPD }\end{array}$ & $\begin{array}{l}\text { Plasma } \\
\text { after BPD }\end{array}$ & $P$ value & $\begin{array}{l}\text { Plasma } \\
\text { before RYGB }\end{array}$ & $\begin{array}{l}\text { Plasma } \\
\text { after RYGB }\end{array}$ & $P$ value & $\begin{array}{l}\text { Plasma } \\
\text { TRVL glucose } \\
\text { administration }\end{array}$ & $\begin{array}{l}\text { Plasma } \\
\text { gastric glucose } \\
\text { administration }\end{array}$ & $P$ value \\
\hline \multicolumn{10}{|c|}{ Primary hepatocyte cultures } \\
\hline $\begin{array}{l}\text { G6Pase } \\
\text { (relative expression) }\end{array}$ & $25.96 \pm 3.30$ & $12.39 \pm 0.67$ & 0.045 & $23.39 \pm 3.78$ & $22.66 \pm 3.91$ & NS & $23.35 \pm 2.30$ & $8.27 \pm 0.90$ & 0.006 \\
\hline $\begin{array}{l}\text { Akt Ser473/total Akt } \\
\text { (arbitrary units) }\end{array}$ & $0.60 \pm 0.03$ & $1.64 \pm 0.14$ & 0.006 & $0.58 \pm 0.03$ & $0.63 \pm 0.03$ & NS & $1.32 \pm 0.22$ & $2.65 \pm 0.20$ & 0.016 \\
\hline $\begin{array}{l}\text { GLUT4/BAct } \\
\text { (arbitrary units) }\end{array}$ & $0.72 \pm 0.03$ & $1.68 \pm 0.15$ & 0.002 & $0.70 \pm 0.06$ & $0.73 \pm 0.07$ & NS & $0.83 \pm 0.08$ & $2.27 \pm 0.33$ & 0.016 \\
\hline $\begin{array}{l}\text { 2-DG6P } \\
\text { (pmol) }\end{array}$ & $12.35 \pm 1.27$ & $86.24 \pm 6.39$ & 0.002 & $13.12 \pm 1.28$ & $13.66 \pm 0.75$ & NS & $9.88 \pm 0.91$ & $71.18 \pm 5.48$ & 0.001 \\
\hline
\end{tabular}

Data are expressed as mean \pm SEM.

BPD, biliopancreatic diversion; 2-DG6P, 2-deoxyglucose-6-phosphate; GLUT4, glucose transporter 4; G6Pase, glucose 6-phosphatase; PEPCK1, phosphoenolpyruvate carboxykinase 1; RYGB, Roux-en-Y gastric bypass; TRVL, Thiry-Vella loop. 
A

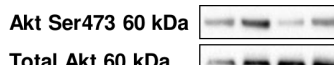

C
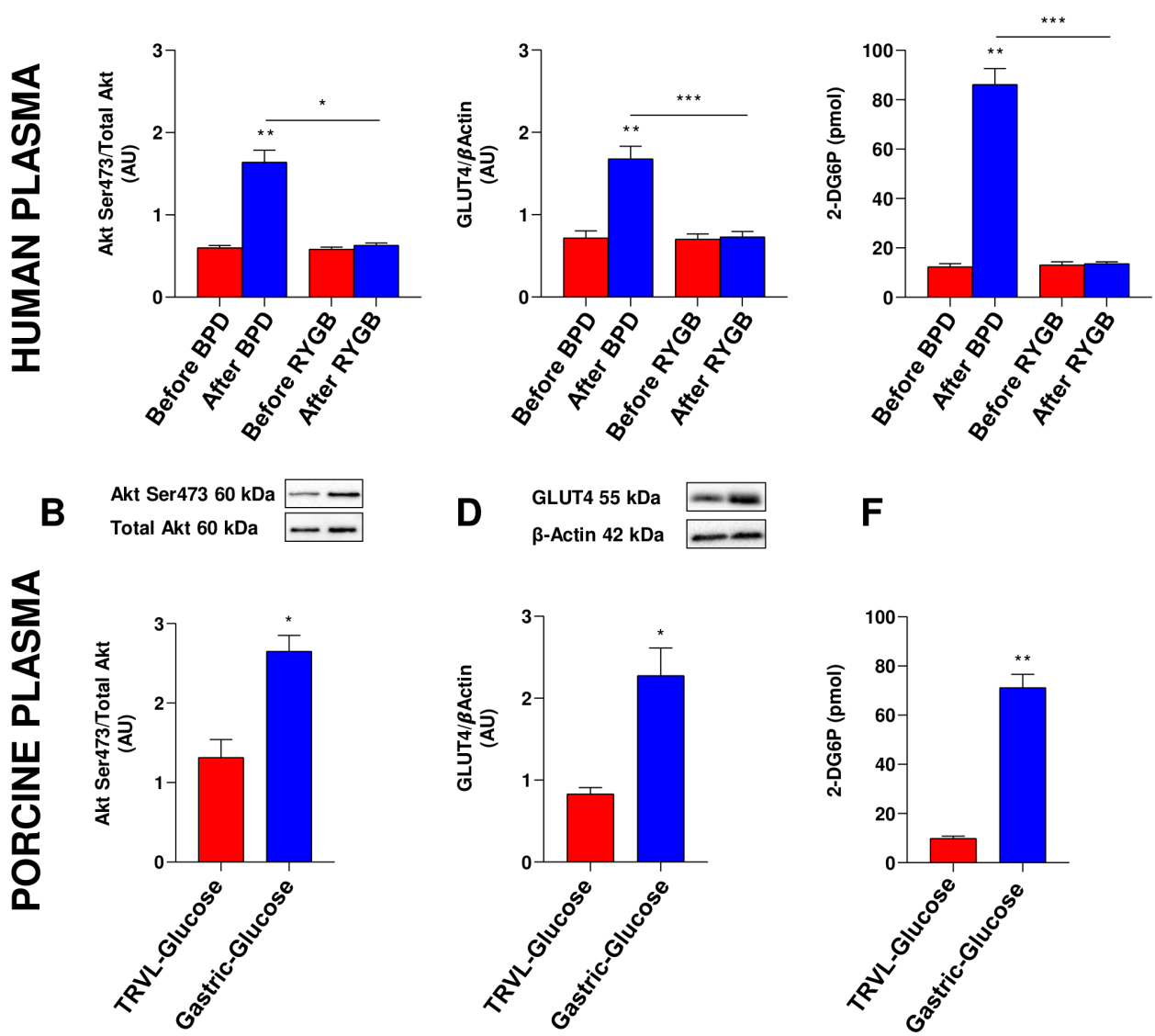

\section{$\mathbf{F}$}

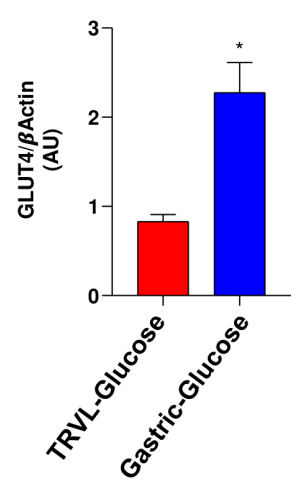

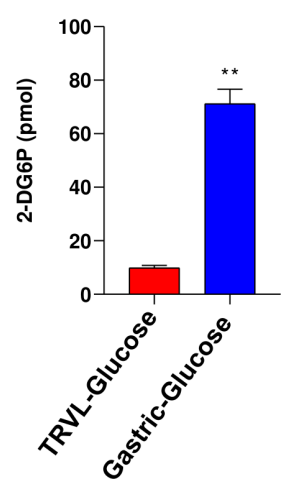

Figure 6 Insulin signalling and GLUT4 translocation in human primary myoblast. (A,B) Akt Ser473 phosphorylation was significantly increased after stimulation of primary myoblast cultures with plasma from subjects who underwent BPD (A) and during gastric glucose administration following jejunectomy in pigs (B). (C,D) GLUT4 expression in primary myoblast cultures was significantly increased after stimulation with plasma from subjects who underwent $B P D(C)$ and during gastric glucose administration following jejunectomy in pigs $(D)$. $(E, F)$ Glucose uptake was significantly increased after stimulation of primary myoblast cultures with plasma from subjects who underwent $B P D(E)$ and during gastric glucose administration following jejunectomy in pigs (F). ${ }^{*} \mathrm{P}<0.02$; ${ }^{* *} \mathrm{P}<0.006$; ${ }^{* *}{ }^{*} \mathrm{P}<0.0005$. BPD, biliopancreatic diversion; 2-DG6P, 2-deoxyglucose-6-phosphate; GLUT4, glucose transporter 4; RYGB, Roux-en-Y gastric bypass; TRVL, Thiry-Vella loop.

One of the biological functions of Akt is its role on insulinmediated GLUT4 translocation to plasma membrane of skeletal muscle cells. ${ }^{31}$ Therefore, we investigated GLUT4 expression and membrane distribution as well as glucose uptake in myoblasts incubated with insulin and plasma of subjects undergoing MS or pig plasma.

Both plasma from subjects undergoing BPD (table 3 ) and plasma from pigs during gastric glucose load (table 3) increased GLUT4 expression (figure 6C,D) and glucose uptake (figure 6E,F). No significant difference was found after stimulation with plasma of RYGB participants.

These data support the role of jejunal exclusion in the greater improvement of whole-body insulin sensitivity observed after BPD.

\section{DISCUSSION}

Overall, our study shows that the jejunum plays a crucial role in controlling insulin sensitivity in both animals and humans through a distinctive metabolic signature involving increased gluconeogenetic metabolites.
To prove that the jejunum is central in the onset of insulin resistance, we performed jejunectomy in pigs and made a jejunal loop with intact nerve and vessel connections. We administered a glucose load in the stomach and infused glucose tracers 1 week after jejunectomy and, in a different day, the glucose load was injected in the jejunal-loop. Infusion of glucose in the jejunalloop worsened insulin sensitivity as compared with intragastric administration following jejunectomy.

The plasma of subjects before MS obtained during OGTT as well as plasma taken during TRVL glucose stimulation increased gluconeogenesis in hepatocyte and impaired insulin signalling in myocytes in vitro.

While hepatic insulin sensitivity ameliorated after both types of surgical operations, peripheral insulin sensitivity, that is, the one involving muscle and adipose tissue, was increased early only after BPD, and insulin secretion was consensually reduced as a mechanism of compensation for the reduced degree of tissue insulin resistance. In contrast, the total ISR in response to the oral glucose challenge was increased at 1 week after RYGB.

An exaggerated release of GLP1 associated with insulin hypersecretion has been extensively observed ${ }^{32-34}$ after RYGB. The 


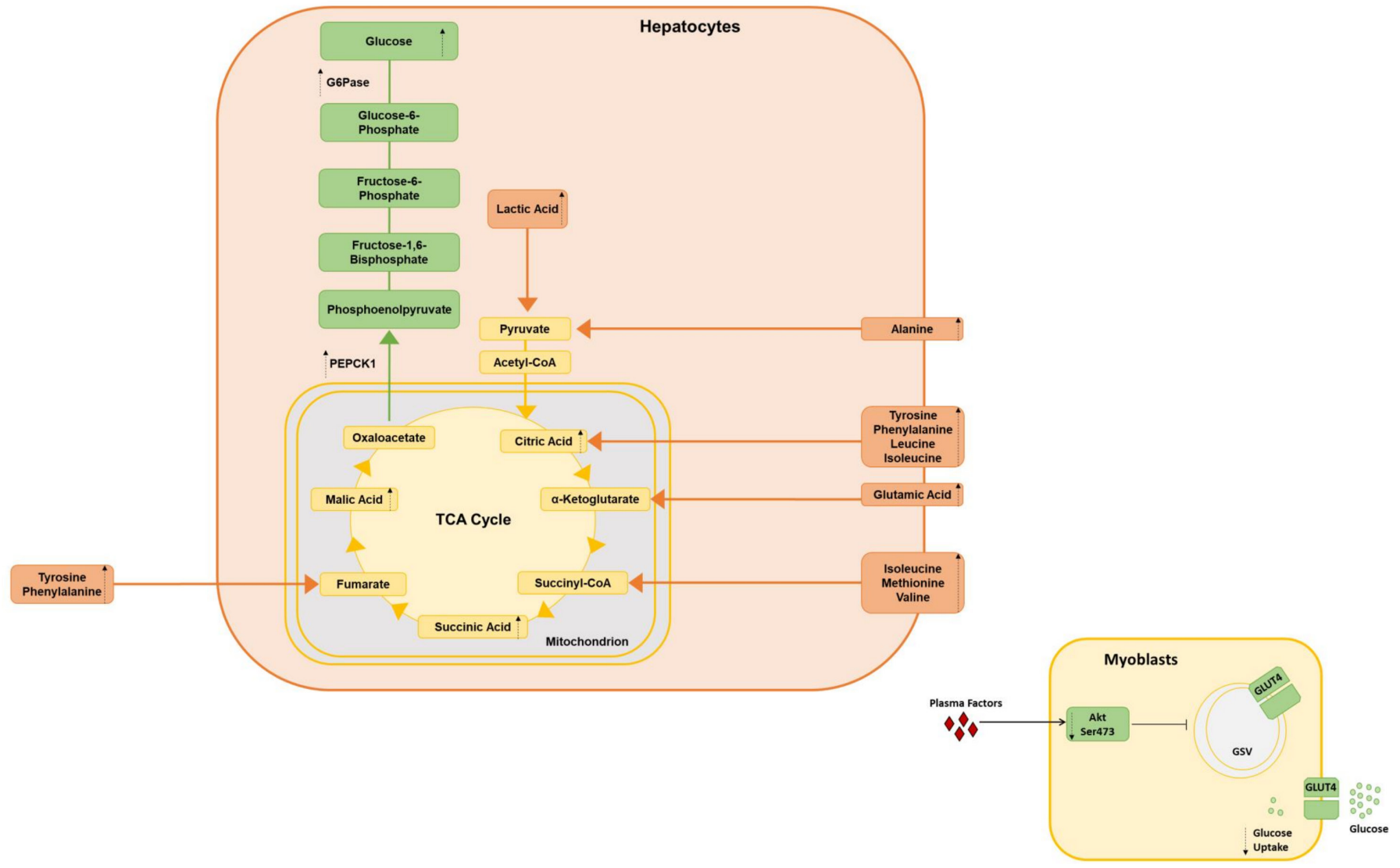

Figure 7 Metabolic pathways related to the metabolites identified in human and pig plasma. The figure shows plasma metabolites that were higher when glucose was administered before BPD as well as in the Thiry-Vella loop as compared with post-BPD or gastric glucose administration following jejunectomy conditions. Some of these metabolites are gluconeogenetic precursors (in orange) promoting liver gluconeogenesis through increased expression of PEPCK1 and G6Pase. The gluconeogenesis pathway and the TCA are schematically represented. Plasma factor/s impair Akt Ser473 phosphorylation in the muscle and thus reduce the translocation of GLUT4 storage vesicles and glucose uptake. BPD, biliopancreatic diversion; TCA, tricarboxylic acid cycle.

early phase of postprandial GLP-1 secretion seems to be mediated by $\mathrm{L}$ cells populating the duodenum ${ }^{35}$ via the activation of sodium-glucose cotransporter 1 , a sensor linking glucose to incretin release. ${ }^{36}$ Glucose triggers GLP1 secretion in proportion to its luminal concentration; hence, GLP1 secretion is stimulated more in the duodenum were the levels of glucose are the highest; half of all duodenal L cells in humans are, in fact, activated acutely by intraduodenal glucose infusion. ${ }^{37}$ The gastric remnant in the classic Scopinaro's BPD is $400-600 \mathrm{~mL}$ that, compared with the $30 \mathrm{~mL}$ gastric pouch in RYGB, means a 10-20 times larger gastric reservoir. This anatomical characteristic can explain the slower glucose release in the proximal gut after BPD than after RYGB and the different effect on GLP1 secretion.

The increased GLP-1 circulating levels after RYGB might also explain the improved hepatic insulin resistance and reduced gluconeogenesis reported in the literature. In fact, GLP-1 (9-36) amide infusion induces $50 \%$ suppression of hepatic glucose production. ${ }^{38}$ However, it does not act directly on hepatocytes, but rather on the central nervous system that regulates hepatic glucose metabolism. ${ }^{36}$ In contrast, duodenal-jejunal bypass in rats is associated with a significant reduction of hepatic gluconeogenetic enzymes PEPCK1 and G6Pase, ${ }^{39}$ suggesting a direct effect of circulating factors on the liver.

Peripheral insulin sensitivity improves after RYGB in proportion to weight $\operatorname{loss}^{40}$; accordingly, we did not find a significant improvement of whole-body insulin sensitivity at 1 week after surgery nor a direct effect of plasma from subjects operated of RYGB on myocytes to improve insulin signalling. In contrast, we observed a significant improvement of both hepatic and peripheral insulin sensitivity after BPD, as well as a direct action of human plasma on myocyte insulin signalling.

Our results are in agreement with the findings of GarridoSanchez et $a l,{ }^{41}$ who compared the effects of Scopinaro's BPD with SG showing a significant improvement of insulin sensitivity already at 15 days after BPD.

Further supporting our hypothesis, we found that the administration of glucose in the TRVL impaired insulin sensitivity without any correlation with GLP-1 levels.

Metabolomics analysis reveals a similar pattern of expression of several metabolites in both humans and pigs. Indeed, bile acids and proline increased particularly after BPD and in the jejunectomy experimental condition, whereas other metabolites, like gluconeogenetic substrates (succinic acid, lactic acid, glutamic acid and alanine) as well as BCAAs, decreased (figure 7) more after BPD than after RYGB.

Several studies report an increase in circulating levels of BCAAs in subjects with obesity and insulin resistance. ${ }^{42-44}$ Accordingly, 4 weeks of dietary reduction of BCAAs in subjects affected by T2D decrease insulin secretion and increase postprandial insulin sensitivity. $^{45}$ 
In vitro and in vivo studies demonstrated that BCAAs activate the mammalian target of rapamycin/ribosomal protein S6 kinase $\beta-1$ kinase pathway and, consequently, the phosphorylation of insulin receptor-substrate-1, leading to insulin resistance. ${ }^{46} 47$ In addition, a higher BCAA catabolic flux induces glucose intolerance by increasing gluconeogenesis through the transamination of glutamate to alanine, a major gluconeogenetic precursor. ${ }^{48}$

In our study, BCAAs, alanine and glutamic acids were elevated before MS and after jejunal loop stimulation, further supporting the idea that jejunal stimulation contribute to insulin resistance by increasing the gluconeogenesis rate. In fact, an important factor controlling gluconeogenesis is the substrate availability. In primary mouse hepatocytes, glycerol induces expression of G6Pase, the key terminal enzyme in gluconeogenesis. ${ }^{49}$

After MS and in the jejunectomy experimental model, we observed a reduction of gluconeogenetic substrates and an increase of proline and 5-oxoproline. Increased levels of proline and 5-oxoproline indicate an increased catabolism of glutamic acid. In fact, proline derives from the catabolism of glutamine formed, in turn, from the hydrolysis of glutamic acid via the action of glutaminase, while 5-oxoproline is a cyclised derivative of glutamic acid. ${ }^{50}$

The increase of circulating gluconeogenetic substrates in TRVL model was associated with the observed higher EGP per unit of insulin. The liver is the major contributor to the production of endogenous glucose that is achieved by glycogen breakdown as well as by de novo glucose synthesis from available precursors. Increased rates of EGP, as observed in patients with T2D, contribute to hyperglycaemia and worsened glycaemic control.

The mechanism of action of the jejunum in hepatic and peripheral insulin resistance was confirmed by our in vitro studies. We found that plasma obtained during glucose stimulation of the jejunum in pigs and during OGTT preceding surgery increased PEPCK1 as well as G6Pase mRNA expression in primary hepatocyte cultures. This suggests that the plasma contained substance/s counteracting the action of insulin and stimulating PEPCK1 and G6Pase, the rate-limiting enzymes for gluconeogenesis.

Glutamine, lactate and tricarboxylic acid cycle intermediates are glucose precursors in the gluconeogenesis pathway. PEPCK1 decarboxylates and phosphorylates oxaloacetate to form phosphoenolpyruvate, while G6Pase catalyses the hydrolysis of glucose-6-phosphate to glucose (figure 7). Therefore, these enzymes facilitate cataplerosis to generate glucose in the TRVL and in obesity in humans. Instead, jejunectomy in pigs and jejunal bypass in humans act inversely, amplifying the antigluconeogenetic action of insulin.

The plasma collected during the glucose stimulation of jejunum in pigs and during the OGTT before surgery inhibited glucose uptake and GLUT4 expression in myoblasts. The opposite happened with plasma drawn during intragastric glucose challenge in pigs after jejunectomy and in humans after jejunal bypass.

Our results suggest that the jejunum produces factor/s counteracting the action of insulin. Recently, we have shown that the degree of insulin sensitivity depends on the route of glucose administration ${ }^{51}$ : oral glucose administration leading to increased insulin secretion and compensatory insulin resistance as compared with the intravenous route of glucose administration.

We acknowledge some limitations of our study. The difference between the deproteinated plasma used for metabolomics and the unadulterated plasma used in cell culture studies means that there may be other factors, including proteins/peptides in the plasma that are not accounted for but could be of potential importance. In this respect, the metabolomic profile may have some upstream regulators which could be the protagonists in the in vitro effect and that elude identification in this study. In conclusion, we show that the jejunum plays a major role in inducing insulin resistance and that its bypass ameliorates insulin sensitivity. In clinical practice, BPD may be superior to RYGB with respect to improving glycaemic control in patients with marginal endocrine pancreatic function at baseline as it is not reliant on boosting insulin secretion. Our results lay the foundation for the discovery of gut molecular mechanisms implicated in the pathophysiology of insulin resistance and, thus, to new possible drugs for the treatment of type 2 diabetes mimicking the effects of MS.

\section{Author affiliations}

${ }^{1}$ Fondazione Policlinico Universitario A. Gemelli IRCCS, Rome, Italy

${ }^{2}$ Università Cattolica del Sacro Cuore, Rome, Italy

${ }^{3}$ Department of Computer, Control, and Management Engineering "Antonio Ruberti", Universityof Rome "Sapienza", Rome, Italy

${ }^{4}$ Department of Surgical Sciences, Sapienza University of Rome, Rome, Italy ${ }^{5}$ CNR-Institute of Systems Analysis and Computer Science (IASI), Rome, Italy ${ }^{6}$ Department of Surgery, Azienda Ospedaliera S. Camillo Forlanini, Rome, Italy ${ }^{7}$ Department of Molecular and Clinical Medicine, Institute of Medicine, the Sahlgrenska Academy at the University of Gothenburg, Gothenburg, Sweden

${ }^{8}$ Cardiometabolic Risk Laboratory, Institute of Clinical Physiology, CNR, Pisa, Italy

${ }^{9}$ Division of Diabetes \& Nutritional Sciences, Faculty of Life Sciences \& Medicine,

King's College London, London, UK

${ }^{10}$ Department of Medicine III, Universitätsklinikum Carl Gustav Carus an der Technischen, Universität Dresden, Dresden, Germany

Twitter Giulia Angelini @GiuliaAngelini7 and Ivo Boskoski @ivoboskoski

Acknowledgements We thank Mrs Anna Caprodossi for her technical assistance. Contributors GM and GA designed the study. SS and AB performed the analysis of the data. GA and SA performed the study and acquired the data. LC-G, JC-M, IB, PLM, MR and GC performed the surgical procedures. AG and MG measured stable isotopes and revised the article. GM and GA wrote the manuscript. SB and GC revised and improved the manuscript.

Funding This study was founded by an internal grant from the Catholic University. Competing interests None declared.

Patient and public involvement Patients and/or the public were not involved in the design, conduct, reporting or dissemination plans of this research.

Patient consent for publication Not required.

Ethics approval The protocol was approved by the ethical committee of the Catholic University of Rome, Italy.

Provenance and peer review Not commissioned; externally peer reviewed.

Data availability statement Data are available upon reasonable request. The datasets generated during the current study are available from the corresponding author on reasonable request.

\section{ORCID iDs}

Giulia Angelini http://orcid.org/0001-6753-745X

Ivo Boskoski http://orcid.org/0000-0001-8194-2670

Amalia Gastaldelli http://orcid.org/0000-0003-2594-1651

Geltrude Mingrone http://orcid.org/0000-0003-2021-528X

\section{REFERENCES}

1 Schauer PR, Bhatt DL, Kirwan JP, et al. Bariatric surgery versus intensive medical therapy for diabetes - 5-year outcomes. N Engl J Med 2017;376:641-51.

2 Mingrone G, Panunzi S, De Gaetano A, et al. Bariatric surgery versus conventional medical therapy for type 2 diabetes. N Engl J Med 2012;366:1577-85.

3 Ikramuddin S, Korner J, Lee W-J, et al. Roux-En-Y gastric bypass vs intensive medical management for the control of type 2 diabetes, hypertension, and hyperlipidemia: the diabetes surgery study randomized clinical trial. JAMA 2013;309:2240-9.

4 Mingrone G, Panunzi S, De Gaetano A, et al. Bariatric-metabolic surgery versus conventional medical treatment in obese patients with type 2 diabetes: 5 year follow-up of an open-label, single-centre, randomised controlled trial. Lancet 2015;386:964-73.

5 Schauer PR, Kashyap SR, Wolski K, et al. Bariatric surgery versus intensive medical therapy in obese patients with diabetes. N Engl J Med 2012;366:1567-76.

6 Ikramuddin S, Korner J, Lee W-J, et al. Lifestyle intervention and medical management with vs without Roux-en-Y gastric bypass and control of hemoglobin A1c, LDL 
cholesterol, and systolic blood pressure at 5 years in the diabetes surgery study. JAMA 2018:319:266-78.

7 Inge TH, Courcoulas AP, Jenkins TM, et al. Five-Year outcomes of gastric bypass in adolescents as compared with adults. N Engl J Med 2019;380:2136-45.

8 DeFronzo RA, Tripathy D. Skeletal muscle insulin resistance is the primary defect in type 2 diabetes. Diabetes Care 2009;32 Suppl 2:S157-63.

9 Guidone C, Manco M, Valera-Mora E, et al. Mechanisms of recovery from type 2 diabetes after malabsorptive bariatric surgery. Diabetes 2006:55:2025-31.

10 Mingrone G, Castagneto-Gissey L. Type 2 diabetes mellitus in 2013: a central role of the gut in glucose homeostasis. Nat Rev Endocrino/ 2014;10:73-4.

11 Chambers AP, Jessen L, Ryan KK, et al. Weight-independent changes in blood glucose homeostasis after gastric bypass or vertical sleeve gastrectomy in rats. Gastroenterology 2011;141:950-8.

12 Cummings DE, Overduin J, Shannon MH, et al. Hormonal mechanisms of weight loss and diabetes resolution after bariatric surgery. Surg Obes Relat Dis 2005;1:358-68.

13 Wilson-Pérez HE, Chambers AP, Ryan KK, et al. Vertical sleeve gastrectomy is effective in two genetic mouse models of glucagon-like peptide 1 receptor deficiency. Diabetes 2013;62:2380-5.

14 Patti M-E, Houten SM, Bianco AC, et al. Serum bile acids are higher in humans with prior gastric bypass: potential contribution to improved glucose and lipid metabolism. Obesity 2009;17:1671-7.

15 Harris L-A, Kayser BD, Cefalo C, et al. Biliopancreatic diversion induces greater metabolic improvement than Roux-en-Y gastric bypass. Cell Metab 2019;30:855-64.

16 Baud G, Daoudi M, Hubert T, et al. Bile diversion in Roux-en-Y gastric bypass modulates sodium-dependent glucose intestinal uptake. Cell Metab 2016;23:547-53.

17 Rubino F, Gagner M, Gentileschi P, et al. The early effect of the Roux-en-Y gastric bypass on hormones involved in body weight regulation and glucose metabolism. Ann Surg 2004;240:236-42.

18 Saeidi N, Meoli L, Nestoridi E, et al. Reprogramming of intestinal glucose metabolism and glycemic control in rats after gastric bypass. Science 2013;341:406-10.

19 Cavin J-B, Couvelard A, Lebtahi R, et al. Differences in alimentary glucose absorption and intestinal disposal of blood glucose after Roux-en-Y gastric bypass vs sleeve gastrectomy. Gastroenterology 2016;150:454-64.

20 Casella-Mariolo J, Castagneto-Gissey L, Angelini G, et al. Simulation of gastric bypass effects on glucose metabolism and non-alcoholic fatty liver disease with the Sleeveballoon device. EBioMedicine 2019;46:452-62.

21 Salinari S, le Roux CW, Bertuzzi A, et al. Duodenal-jejunal bypass and jejunectomy improve insulin sensitivity in Goto-Kakizaki diabetic rats without changes in incretins or insulin secretion. Diabetes 2014;63:1069-78.

22 Rubino F, Schauer PR, Kaplan LM, et al. Metabolic surgery to treat type 2 diabetes: clinical outcomes and mechanisms of action. Annu Rev Med 2010;61:393-411.

23 Jackness C, Karmally W, Febres G, et al. Very low-calorie diet mimics the early beneficial effect of Roux-en-Y gastric bypass on insulin sensitivity and $\beta$-cell function in type 2 diabetic patients. Diabetes 2013;62:3027-32.

24 Salinari S, Carr RD, Guidone C, et al. Nutrient infusion bypassing duodenum-jejunum improves insulin sensitivity in glucose-tolerant and diabetic obese subjects. Am J Physiol Endocrinol Metab 2013;305:E59-66.

25 Dalla Man C, Yarasheski KE, Caumo A, et al. Insulin sensitivity by oral glucose minima models: validation against clamp. Am J Physiol Endocrinol Metab 2005;289:E954-9.

26 Breda E, Cavaghan MK, Toffolo G, et al. Oral glucose tolerance test minimal model indexes of beta-cell function and insulin sensitivity. Diabetes 2001;50:150-8.

27 Jung S-H, Jung C-H, Reaven GM, et al. Adapting to insulin resistance in obesity: role of insulin secretion and clearance. Diabetologia 2018;61:681-7.

28 Matthews DR, Hosker JP, Rudenski AS, et al. Homeostasis model assessment: insulin resistance and beta-cell function from fasting plasma glucose and insulin concentrations in man. Diabetologia 1985;28:412-9.

29 Bojsen-Møller KN, Dirksen C, Jørgensen NB, et al. Increased hepatic insulin clearance after Roux-en-Y gastric bypass. J Clin Endocrinol Metab 2013;98:E1066-71.
30 Wewer Albrechtsen NJ, Kuhre RE, Toräng S, et al. The intestinal distribution pattern of appetite- and glucose regulatory peptides in mice, rats and pigs. BMC Res Notes 2016;9:60

31 Jiang ZY, Zhou QL, Coleman KA, et al. Insulin signaling through Akt/protein kinase B analyzed by small interfering RNA-mediated gene silencing. Proc Natl Acad Sci U S A 2003:100:7569-74.

32 le Roux CW, Aylwin SJB, Batterham RL, et al. Gut hormone profiles following bariatric surgery favor an anorectic state, facilitate weight loss, and improve metabolic parameters. Ann Surg 2006;243:108-14.

33 Laferrère B, Teixeira J, McGinty J, et al. Effect of weight loss by gastric bypass surgery versus hypocaloric diet on glucose and incretin levels in patients with type 2 diabetes. J Clin Endocrinol Metab 2008;93:2479-85.

34 Holst JJ, Madsbad S, Bojsen-Møller KN, et al. Mechanisms in bariatric surgery: gut hormones, diabetes resolution, and weight loss. Surg Obes Relat Dis 2018;14:708-14

35 Theodorakis MJ, Carlson O, Michopoulos S, et al. Human duodenal enteroendocrine cells: source of both incretin peptides, GLP-1 and GIP. Am J Physiol Endocrinol Metab 2006;290:E550-9.

36 Kuhre RE, Frost CR, Svendsen B, et al. Molecular mechanisms of glucose-stimulated GLP-1 secretion from perfused rat small intestine. Diabetes 2015;64:370-82.

37 Sun EW, de Fontgalland D, Rabbitt P, et al. Mechanisms controlling glucose-induced GLP-1 secretion in human small intestine. Diabetes 2017;66:2144-9.

38 Sandoval DA, Bagnol D, Woods SC, et al. Arcuate glucagon-like peptide 1 receptors regulate glucose homeostasis but not food intake. Diabetes 2008:57:2046-54.

39 Kim M, Son YG, Kang YN, et al. Changes in glucose transporters, gluconeogenesis, and circadian clock after duodenal-jejunal bypass surgery. Obes Surg 2015;25:635-41

40 Dirksen C, Jørgensen NB, Bojsen-Møller KN, et al. Mechanisms of improved glycaemic control after Roux-en-Y gastric bypass. Diabetologia 2012;55:1890-901.

41 Garrido-Sanchez L, Murri M, Rivas-Becerra J, et al. Bypass of the duodenum improves insulin resistance much more rapidly than sleeve gastrectomy. Surg Obes Relat Dis 2012:8:145-50.

42 Newgard CB. Interplay between lipids and branched-chain amino acids in development of insulin resistance. Cell Metab 2012;15:606-14.

43 Newgard CB, An J, Bain JR, et al. A branched-chain amino acid-related metabolic signature that differentiates obese and lean humans and contributes to insulin resistance. Cell Metab 2009;9:311-26.

44 Wang TJ, Larson MG, Vasan RS, et al. Metabolite profiles and the risk of developing diabetes. Nat Med 2011;17:448-53.

45 Karusheva Y, Koessler T, Strassburger K, et al. Short-Term dietary reduction of branched-chain amino acids reduces meal-induced insulin secretion and modifies microbiome composition in type 2 diabetes: a randomized controlled crossover trial. Am J Clin Nutr 2019:110:1098-107.

46 Tremblay $\mathrm{F}$, Lavigne $\mathrm{C}$, Jacques $\mathrm{H}$, et al. Role of dietary proteins and amino acids in the pathogenesis of insulin resistance. Annu Rev Nutr 2007:27:293-310.

47 Krebs M, Brunmair B, Brehm A, et al. The mammalian target of rapamycin pathway regulates nutrient-sensitive glucose uptake in man. Diabetes 2007;56:1600-7.

48 Vozarova B, Stefan N, Lindsay RS, et al. High alanine aminotransferase is associated with decreased hepatic insulin sensitivity and predicts the development of type 2 diabetes. Diabetes 2002;51:1889-95.

49 Kalemba KM, Wang Y, Xu H, et al. Glycerol induces G6pc in primary mouse hepatocytes and is the preferred substrate for gluconeogenesis both in vitro and in vivo. J Biol Chem 2019;294:18017-28.

50 Lee WJ, Hawkins RA, Peterson DR, et al. Role of oxoproline in the regulation of neutral amino acid transport across the blood-brain barrier. J Biol Chem 1996;271:19129-33.

51 Mingrone G, Panunzi S, De Gaetano A, et al. Insulin sensitivity depends on the route of glucose administration. Diabetologia 2020;63:1382-95. 